\title{
Quark and lepton mass matrices from localization in M-theory on $G_{2}$ orbifold
}

\author{
Eric Gonzalez, ${ }^{1, *}$ Gordon Kane $\odot,{ }^{1, \dagger}$ Khoa Dang Nguyen $\odot,{ }^{2, \ddagger}$ and Malcolm J. Perry ${ }^{3,4, \S}$ \\ ${ }^{1}$ Leinweber Center for Theoretical Physics, Department of Physics, University of Michigan, \\ Ann Arbor, Michigan 48109, USA \\ ${ }^{2}$ Department of Mathematics, University of Michigan, Ann Arbor, Michigan 48109, USA \\ ${ }^{3}$ DAMTP, Cambridge University, Centre for Mathematical Sciences, \\ Wilberforce Road, Cambridge CB3 OWA, United Kingdom \\ ${ }^{4}$ Department of Physics, Queen Mary University of London, \\ Mile End Road, Bethnal Green, London E1 4NS, United Kingdom
}

(Received 2 February 2021; accepted 4 June 2021; published 28 June 2021)

\begin{abstract}
M-theory compactified on a $G_{2}$ manifold with resolved $E_{8}$ singularities realizes four-dimensional $\mathcal{N}=1$ supersymmetric gauge theories coupled to gravity with three families of Standard Model fermions. Beginning with one $E_{8}$ singularity, three fermion families emerge when $E_{8}$ is broken by geometric engineering deformations to a smaller subgroup with equal rank. In this paper, we use the local geometry of the theory to explain the origin of the three families and their mass hierarchy. We linearize the blowing up of two-cycles associated with resolving $E_{8}$ singularities. After imposing explicit constraints on the effectively stabilized moduli, we arrive at Yukawa couplings for the quarks and leptons. We fit the high scale Yukawa couplings approximately which results in the quark masses agreeing reasonably well with the observations, implying that the experimental hierarchy of the masses is achievable within this framework. The hierarchy separation of the top quark from the charm and up is a stringy effect, while the spitting of the charm and up also depends on the Higgs sector. The Higgs sector cannot be reduced to having a single vacuum expectation value (VEV); all three VEVs must be nonzero. Three extra $U(1)$ s survive to the low scale but are not massless, so $Z$ states are motivated to occur in the spectrum, but may be massive.
\end{abstract}

DOI: $10.1103 /$ PhysRevD.103.126027

\section{INTRODUCTION}

M-theory has been met with considerable success [1-4]. One prediction of compactified M-theory is the existence of $\mathcal{N}=1$ supersymmetry and its soft breaking via gluino condensation, while simultaneously stabilizing all moduli $[4,5]$. M-theory accommodates radiative electroweak symmetry breaking [3], baryogenesis [6], a solution to the strong $C P$ problem [7], and a mechanism for inflation [8]. Last, this framework can include a wide variety of hidden sector dark matter candidates and predict a supergravity spectrum semiqualitatively [3,4]. Moreover, most results from string theories can be extrapolated to M-theory through duality.

\footnotetext{
*ericgz@umich.edu

†kane@umich.edu

*kdng@umich.edu

${ }^{\S}$ malcolm@damtp.cam.ac.uk
}

Published by the American Physical Society under the terms of the Creative Commons Attribution 4.0 International license. Further distribution of this work must maintain attribution to the author(s) and the published article's title, journal citation, and DOI. Funded by SCOAP.
In this paper we focus on an M-theory calculation of the quark and charged lepton masses. The first step is to find an appropriate reduction from eleven to four dimensions. Suppose that spacetime is a product $\mathbb{R}^{3,1} \times X$ where $X$ is a compact seven-dimensional manifold roughly Planck scale in size. Gauge coupling unification and M-theory compactification hint at unbroken supersymmetry at the unification scale. Bergers theorem [9] requires that $\mathcal{N}=1$ SUSY implies that the holonomy group of the manifold $X$ is $G_{2}$. The resultant low-energy theory can only contain $U(1)$ gauge fields. Such a compactification scheme is unrealistic since the Standard Model (SM) contains nonAbelian gauge fields. One introduces singularities into $X$ to ameliorate this issue. A special type of singularity called $\mathrm{ADE}^{1}$ allows non-Abelian gauge groups to exist in the theory. Suppose that the local model of $X$ with ADE singularity is of the form $\mathbb{C}^{2} / \Gamma \times \mathbb{R}^{3}$, where $\Gamma$ is a finite subgroup of $S U(2)$ (see Table 2). Under these circumstances, a super Yang-Mills $\mathcal{N}=1$ multiplet with gauge group $G=S U(k), S O(2 k), E_{6}, E_{7}$, and $E_{8}$, respectively,

\footnotetext{
${ }^{1} \mathrm{ADE}$ stands for $\mathrm{A}, \mathrm{D}$, and E Lie algebra.
} 
will be supported. These singularities can be deformed to break the symmetry of the gauge group $G$ to a subgroup of $G$ with equal rank.

We focus on breaking 248 of $E_{8}$ to the SM particle. The matter that survives the symmetry breaking process consists of three multiplets in the 27 representation of $E_{6}$ and none in the $\overline{\mathbf{2 7}}$ representation of $E_{6}{ }^{2}$ [10]. This can explain why there are three and only three families. We explore the aforementioned symmetry breaking pattern by looking to see if a realistic SM theory can descend from a compactified M-theory construction. We calculate the Yukawa couplings under the assumption that everything originates from a deformed $E_{8}$ theory where the singularity is resolved into a lesser ranked singularity which is associated with the $S U(3) \times S U(2) \times U(1) \times U(1)^{4}$ gauge group. ${ }^{3}$

To explain the origin of the three families and their mass hierarchy, breaking $E_{8}$ to the SM by the traditional Higgs mechanism has been unsuccessful and has shown a lack of predictability, while geometrically engineered M- and F-theories with $E_{8}$ points offer an alternative method of symmetry breaking. Moreover, the authors of [11] and related works suggest M-theory based on an $\hat{E}_{8}-A L E$ space provides more predictability than the analogous model in F-theory [12-14]. Finally, a description of a singular $G_{2}$ manifold with Higgs bundles provides a formulation which makes explicit computation of Yukawa couplings possible $[15,16]$.

We are interested in explicitly calculating the hierarchy of quark mass matrices. As that would include an explicit method for computing matter content, in gauge symmetry breaking through deformation, and their coupling constants, the results would be applicable to a wider study of other matter interaction. We also compute the mass matrix for charged leptons.

The paper is aimed at a wider audience, so some technical details are omitted and referred to as external sources. Section II contains a brief review of M-theory on a $G_{2}$ manifold. Section III describes the resolution of ADE singularities and the method for computing gauge group symmetry breaking. In Sec. IV we explicitly compute this breaking for the $E_{8}$ singularity with an explicit example of how to compute and locate the fermions on $M_{3}$. Section $\mathrm{V}$ discusses the general computation for the Yukawa couplings in a local model which leads to explicit quark and lepton terms in Sec. VI. After some gauge fixing for basespace $M_{3}$ 's parameters, numerical results are discussed in Sec. VIII. We see that the physical hierarchy is achievable with a very small set of solutions, putting a stringent

\footnotetext{
${ }^{2}$ Bourjaily et al. [10] explained that the net number of chiral zero modes was one. So, either 27 or $\overline{\mathbf{2 7}}$ was a normalizable zero mode, but not both. As a convention, we pick the normalizable zero mode to be in 27.

${ }^{3}$ We separate one $U(1)$ factor out to emphasize the SM gauge group.
}

constraint on the moduli of the theory. Section IX discusses the roles of both Yukawa couplings and Higgs vacuum expectation values (VEVs) in this hierarchy.

\section{A BRIEF BACKGROUND OF M-THEORY ON $G_{2}$ SINGULAR MANIFOLDS}

M-theory is an 11-dimensional theory that can be compactified on a compact seven-dimensional (7D) manifold $X$ while the remaining noncompact four dimensions are the classical 4 spacetime. In the supergravity limit, $X$ is necessarily a $G_{2}$ manifold. Moreover, charged chiral particles are only possible on a singular $G_{2}$ manifold [9]. The simplest local model for such a 7D manifold is given by the fibering of $\mathbb{C}^{2} \widehat{\Gamma_{\mathrm{ADE}}}$ over the base $M_{3}$. Here, $M_{3}$ is an associative three-cycle ${ }^{4}$ in the $G_{2}$ manifold. $\Gamma_{\mathrm{ADE}}$ is a finite subgroup of $S U(2)$ acting on $\mathbb{C}^{2} . \mathbb{C}^{2} / \Gamma_{\mathrm{ADE}}$ is an asymptotically locally Euclidean manifold (ALE) with $\mathrm{ADE}$ singularity at the origin. $\mathbb{C}^{2} \widehat{\Gamma_{\mathrm{ADE}}}$ denotes any manifold achieved from $\mathbb{C}^{2} / \Gamma_{\mathrm{ADE}}$ by partially smoothing (resolving) the singularity. Locally, the manifold is of the form

$$
\mathbb{R}^{3,1} \times M_{3} \times \mathbb{C}^{2} \widehat{\Gamma_{\mathrm{ADE}}}
$$

Note that globally, the fiber $\mathbb{C}^{2} \widehat{\Gamma_{\mathrm{ADE}}}$ varies along the base $M_{3}$ where the singularity can be smoothed out to different degrees. More details on a recent construction of compact $G_{2}$ manifolds are in [17-20].

\section{A. Gauge group enhancement}

Inherited from supergravity at a low-energy limit, the basic fields are a metric $g$, a three-form potential $C_{3}$, and a gravitino spinor $\Psi$. We will briefly review the essential properties of the fields needed for this paper. More details are discussed in the Appendix A and [15,16,21,22]. From Chern-Simon (CS) terms, $C_{3}$ is integrated over a manifold of the same dimension, i.e., a 3 submanifold of spacetime. Excluding time, this submanifold is 2D spatial. This 2D submanifold is an $M_{2}$ brane. We say $C_{3}$ electrically couples with $M_{2}$ brane. Dimensional reduction of the $C_{3}$ form on the ALE fiber produces $U(1)$ gauge fields

$$
C_{3}=A_{i} \wedge \omega^{i}+\cdots,
$$

where $A_{i}$ 's are one-forms (vector fields) on $\mathbb{R}^{3,1}$ and $\omega^{i}$,s are harmonic two-forms associated with two-cycles of ALE fibers.

The non-Abelian gauge group is produced in a similar manner as in coincident D6-branes in type IIA string theory [23]. In another perspective independent of duality, the

\footnotetext{
${ }^{4}$ Equations of motion require minimal volume, and an associative cycle is a minimal volume cycle.
} 
gauge symmetry at a ADE singularity comes from the symmetry of the differential form under automorphism of the resolved manifold. Explicitly, the two-forms on the resolved manifold can be expressed as an element of the Lie algebra of the associated ADE group. Therefore, under the automorphic map on the resolved manifold, the form can be transformed under the action of the Lie group. At singular points where some cycles shrink to a single point, the forms in the same orbit under the transformation induced from the automorphism of those cycles correspond to the same state, so the transformation is a gauge transformation. For example, a self-contained description for the gauge transformation from $S U(N)$ singularity, i.e., $A_{N-1}$ type, would be summarized in the diagrams below. The $C_{3}$ is decomposed into the basis of the three-forms. In the local description, the basis elements contain components that are two-forms $\alpha_{i}$ on the two-spheres $\mathbb{C P}^{1}$ which resolves the singularity,

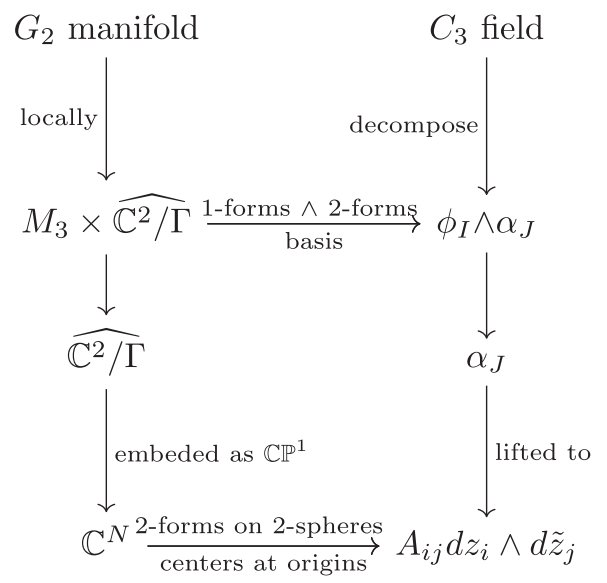

When embedding $\widehat{\mathbb{C}^{2} / \Gamma}$ into $\mathbb{C}^{N}$, we can explicitly write $\alpha_{i}$ in a local coordinate and see the gauge field $A_{i j}$ transforming under the rotations of $S U(N)$. Fibering this on the $M_{3}$ base, we see the corresponding adjoint-valued form $\phi$ mentioned in [16]

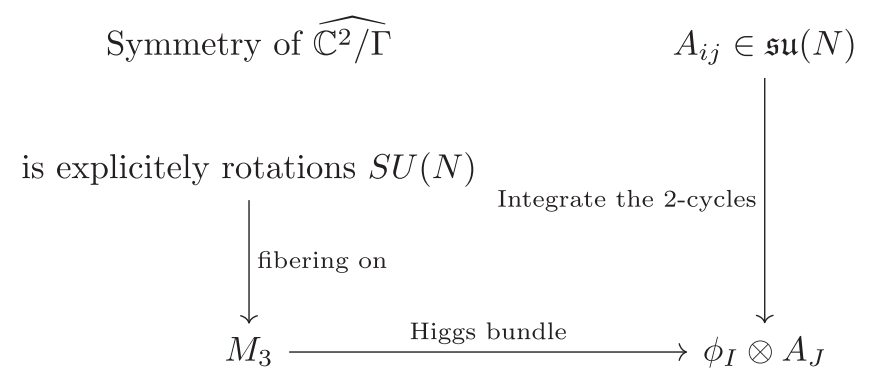

where $\phi \equiv \sum_{I, J} \phi_{I} \otimes A_{J}$ is explicitly a field transform in the adjoint of $S U(N)$ [through $A_{i j} \in \mathfrak{S} \mathfrak{u}(N)$ ], thus befitting the $S U(N)$ gauge description. Similarly, we can embed $D_{N}$, $E_{6}, E_{7}$, and $E_{8}$ type singularities into $\mathbb{R}^{2 N}, \mathbb{C} \otimes \mathbb{O}$ (bioctonions), $\mathbb{U} \otimes \mathbb{O}$ (quateroctonions), and $\mathbb{O} \otimes \mathbb{O}$ (octooctonions), respectively.

The moral of this is the gauge symmetry comes from the geometrical symmetry of $\widehat{\mathbb{C}^{2} / \Gamma}$ which can be explicitly realized by embedding into a covering space. This is an explicit connection to 7D super Yang-Mills theory on $\mathbb{R}^{3,1} \times M_{3}$ by the Higgs bundle. (The connection has been known for a long time through duality without explicit embedding.)

It has always been mentioned that $M_{2}$ branes wrapping ADE singularities will give a non-Abelian gauge. In here, we can see gauge boson $A_{i j}$ explicitly and independently from the duality description.

In a more intuitive sense, the warping of $M_{2}$ branes around nonvanishing ALE cycles creates massive vector bosons. The masses are proportional to the volume of the two-cycles. By shrinking the two-cycles, we are making those massive bosons massless. Moreover, the configuration of the two-cycles (Dynkin diagram) dictates the relation of these bosons and fits them perfectly into a non-Abelian gauge group. Inversely, at any point on $M_{3}$ where the volume of a two-cycle is nonzero, the associated vector boson becomes massive and hence must be removed from the gauge group. Yet, the $U(1)$ in the Cartan subalgebra from (2.2) is unaffected by this, so we still have a $U(1)$ gauge symmetry. Hence, the $n$-ranked gauge group is broken into an $(n-1)$-ranked subgroup and a $U(1)$ (total rank is unchanged). In general, each nonvanishing volume of a basis two-cycle reduces the rank of the group by one and leave a $U(1)$ behind. It is important to note that this is similar to the Higgs mechanism except that the Higgsing happens due to the geometry instead of the traditional Higgs doublets as we will discuss in the next section.

\section{B. Chiral fermion}

On a singularity curve for a non-Abelian gauge group $H$, which is a resolution ${ }^{5}$ of a higher rank singularity of a larger gauge group $G$, chiral fermion solutions are localized at points where the singularity associated with $H$ is worsened by a conical singularity $[1,10,24,25]$. By considering the resulting extra subgroup generated by the extra shrunk twocycles, one can determine the representation of the fermions with respect to the gauge group $H$. We will elaborate this in Sec. IVA.

\section{ADE SINGLARITY, RESOLUTION, AND DEFORMATION}

ADE singularity classifies a family of singularities that has an injective map into the set of general unbroken gauge symmetries in M-theory as a consequence of the Mckay correspondence. Therefore, we briefly review ADE

\footnotetext{
${ }^{5}$ See Sec. III.
} 


Singular point

knot for a $\mathrm{P}^{1}$ and line for an intersection

FIG. 1. An $A_{3}$ type singularity being fully resolved will have a configuration of three Riemann spheres $\mathbb{P}^{1}(\mathbb{C})$ which intersect according to the $A_{3}$ Dynkin diagram. Note that every two spheres intersect at most at one point transversely.

singularity classification. ${ }^{6}$ An ADE singularity can be written as $\mathbb{C}^{2} / \Gamma$ where $\Gamma \subset S U(2)$ is a finite subgroup and acts on $\mathbb{C}^{2}$ by ordinary multiplication. This action has no fixed point other than the origin. Consequently, $\mathbb{C}^{2} / \Gamma$ has a singularity at the origin.

Such a singularity can be made smooth by expanding the singular point into a projective space $\mathbb{P}^{1}(\mathbb{C})$ (topologically just a 2-sphere). This procedure is called "blowing up," and the blown-up space is called a resolution of the original space. However, the blown-up point may not be resolved completely and still have some remaining singular points on the $\mathbb{P}^{1}$. We have to keep blowing up those points until there is no singularity. The result is a collection of $\mathbb{P}^{1}$ 's intersecting of each other. The intersection pattern is exactly the Dynkin diagram of the type of singularity. Figure 1 gives a pictorial illustration of a singularity of type $A_{3}$. Each of the consequent $\mathbb{P}^{1}$ can be called a two-cycle. So, a singularity of type $A_{3}$ is one that, when completely resolved, has a configuration of $A_{3}$. Similarly, a singularity of a certain Dynkin diagram has the blown-up configuration of that diagram. The explicit diagrams with the associated group are in Fig. 2.

We have seen that the two-cycles $\mathbb{P}^{1}$ directly relate to the smoothing of singularities. We can use the volume of the two-cycles to parametrize the resolution. Such a method of smoothly parametrizing the blowing up is called deformation.

For each two-cycle, we use a harmonic one-form $\phi^{7}$ on $M_{3}$, which can be thought of as a metric-invariant threevector field on $M_{3}$, to parametrize the size of the two-cycle. Alternatively, Katz et al. $[11,28]$ use the coefficients in the

\footnotetext{
${ }^{6}$ Originally due to [26]. ALE construction by hyper-Kähler quotients is in [27].

${ }^{7}$ This is in fact the VEV of the Higgs field $\phi$ in 7D Yang-Mills theory we mentioned earlier.
}

FIG. 2. Dynkin diagram and associated groups.

Cartan subalgebra as the parameters. Consistently, there is a one-to-one bijection between the two parametrizations given by Table I. Following the existing literature, we denote $\hat{G}\left(f_{1}, f_{2}, f_{3}, \ldots, f_{n}\right)$ as the family of $\widehat{\mathbb{C}}^{2 / \Gamma_{G}}$ parametrized by the coordinates $f_{i}$ in the Cartan subalgebra where $n$ is the rank of $G$ and use Table I to compute the "volume" one-form $\phi$ when needed. ${ }^{8}$

Note that the theory actually agrees with the distance conjecture from Vafa et al. [30]. The construction of M-theory compactified on a circle is dual to type IIA string theory. Specifically, when a M2 brane wraps around one of the basis two-cycles of the resolved $E_{8}$ singularity in our model, it is dual to a string wrapping around a circle in type IIA. When the moduli in our theory go to infinity, it is equivalent to the volume of the two-cycles going to infinity. This is dual to the infinite radius limit of a circle in type IIA. Vafa et al. [30] have already argued about the infinite tower of massless states in the type IIA side for the infinite radius limit. M-theory inherits this tower through dimensional reduction.

\section{IV. $E_{8}$ BREAKING}

Our goal is to describe all the particles by resolving one single ADE singularity. $E_{8}$ is the only simple Lie group that does the job. $E_{8}$ and its breaking have been studied by several authors [12,15,31-37]. To understand the breaking, we first explicitly write down the simple roots of $E_{8}$ in the Dynkin diagram order (see Table 2) where $e_{i}$ 's are orthogonal vectors in $\mathbb{R}^{n, 1}$. Let $\hat{E}_{8}\left(f_{1}, \ldots, f_{8}\right)$ be the resolution of a $E_{8}$ singularity parametrized by deformation moduli $f_{i}$ 's which are one-forms on $M_{3}$. The simple roots are associated with the volumes of the blown-up two-cycles by Table I [11].

Each simple root, or equivalently each knot on the Dynkin diagram, will initially represent a vanishing cycle at the singularity. To break a group to a smaller group, we will "cut" a knot on their diagram so that we get the

\footnotetext{
${ }^{8}$ More details on the root system and deformation are in [29].
} 
TABLE I. Positive roots of $E_{n}$ and the associated one-forms (sometimes called "area" in literature) controlling the sizes of two-cycles on the ALE fiber. This is Table 1 in [11] with permission.

\begin{tabular}{ccc}
\hline \hline & $\begin{array}{c}\text { Positive roots } \\
\text { of } E_{n}\end{array}$ & $\begin{array}{c}\text { Volume of corresponding } \\
\text { two-cycle }\end{array}$ \\
\hline$e_{i}-e_{j>i}$ & $f_{i}-f_{j>i}$ \\
$n \geq 6$ & $-e_{0}+e_{i}+e_{j}+e_{k}$ & $f_{i}+f_{j}+f_{k}$ \\
$n=8$ & $-2 e_{0}+\Sigma_{j=1}^{6} e_{j}$ & $\Sigma_{j=1}^{6} f_{j}$ \\
\hline \hline
\end{tabular}

diagram of the smaller group. Each "cutting" is performed by blowing up the cycle (which was initially vanishing) associated with the knot. We recall that each cycle in the above Dynkin diagram gives rise to a boson whose mass is proportional to the volume of the cycle. Therefore, a vanishing cycle in the above Dynkin diagram will result in a massless boson. The goal is to keep the SM gauge bosons massless (zero volume cycles) while the other bosons are massive (nonzero volume cycles). We will follow the breaking path ${ }^{9}$ of [32]. Figure 3 summarizes the above steps. In the figure, we start with an $E_{8}$ singularity which corresponds to $\hat{E}_{8}(0,0,0,0,0,0,0,0)$, and then turn on the volumes of the cycles associated with the crossed knots by giving nonzero values for one-form $f_{i}$ 's. There are five volumes needed to be turned on, so we parametrize $f_{i}$ 's by five nonzero one-forms $a, b, c, d$, and $Y$ [note that $Y$ here is the one-form associated with hypercharge $U(1)^{Y}$, not the hypercharge itself]. They are simply parameters that are linearly combined in a specific way so that the volumes of the cycles vanish or blow up appropriately by Table I. Then the final manifold is parametrized as [11]

$$
\begin{gathered}
\hat{E}_{8}\left(a+b+c+d+\frac{2}{3} Y, a-b+c+d+\frac{2}{3} Y,\right. \\
-c-d-\frac{7}{3} Y,-c-d-\frac{7}{3} Y,-c-d+\frac{8}{3} Y, \\
\left.-c-d+\frac{8}{3} Y,-c+3 d-\frac{4}{3} Y, 2 c-2 d-\frac{4}{3} Y\right) .
\end{gathered}
$$

We can check each step of Fig. 3 by setting all $a, b, c, d$, and $Y$ in (4.2) to zero, then turn them on accordingly to each step, and compute the volumes using Table I. In the following, we can check the volumes of the cycles corresponding to the simple roots in the final step

\footnotetext{
${ }^{9}$ Different paths to the same subgroup will lead to the same physics. This is because if there is a diffeomorphism between $X_{1}$ and $X_{2}$ so that their hyper-Kähler structures agree, then they are isometric.
}

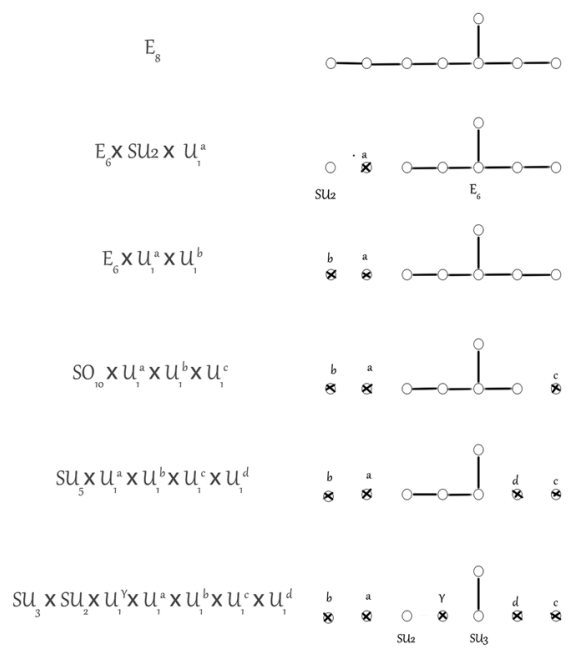

FIG. 3. Breaking of $E_{8}$ by resolving singularity.

$$
\left(\begin{array}{cc}
e_{1}-e_{2} & 2 b \\
e_{2}-e_{3} & a-b+2 c+2 d+3 Y \\
e_{3}-e_{4} & 0 \\
e_{4}-e_{5} & -5 Y \\
e_{5}-e_{6} & 0 \\
e_{6}-e_{7} & -4 d+4 Y \\
e_{7}-e_{8} & -3 c+5 d \\
-e_{0}+e_{6}+e_{7}+e_{8} & 0
\end{array}\right)
$$

This is exactly the configuration of Fig. 3. Note that one can use any different set of one-forms as long as they fulfill the desired configuration and sufficiently parametrize the independent nonvanishing cycles. Therefore, whatever constraint we make to avoid an unwanted shrunk cycle which will lead to an extra massless boson, we have to make nonzero volumes in the above table remain nonzero. This would mean

$$
\begin{gathered}
b \neq 0, \quad a-b+2 c+2 d+3 Y \neq 0, \\
Y \neq 0, \quad Y \neq d, \quad c \neq \frac{5}{3} d .
\end{gathered}
$$

\section{A. Fermion representations}

Given a gauge group $H$ for the theory, the corresponding cycles on the fiber are shrunk everywhere along the base manifold $M_{3}$. Those cycles correspond to the simple roots of $H$. A matter representation happens at the points where additional cycles associated with positive roots (see Table I) vanish. By letting the positive roots vanish one by one, we can find all the resulting representations. We will do a few examples showing how to calculate the representation. First, we consider the $e_{2}-e_{3}$ cycle. Using the above table, we conclude that the associated volume is 
$f_{2}-f_{3}=a-b+2 c+2 d+3 Y$. Now, we consider the curve where this particular cycle vanishes: $a-b+2 c+2 d+3 Y=0$. In order to know what representation emerges at this curve, we consider what kind of weight diagram is generated from $e_{2}-e_{3}$ and the roots from the gauge group (corresponding to the globally shrunk cycles) $e_{3}-e_{4}$ [corresponding to $S U(2)$ ], and $e_{5}-e_{6}$ and $-e_{0}+e_{6}+e_{7}+e_{8}$ [corresponding to $S U(3)$ ]. In more details, we will try to find what are the positive roots we can get from $e_{2}-e_{3}$ by adding or subtracting $e_{3}-e_{4}, e_{5}-e_{6}$, and $-e_{0}+e_{6}+e_{7}+e_{8}$.

$\begin{array}{cc}S U(2) & S U(3) \\ & \text { from adding or substracting } \\ e_{3}-e_{4} & e_{5}-e_{6} \text { or } \\ e_{2}-e_{3} & \left.-e_{0}+e_{6}+e_{7}+e_{8}\right) \\ & e_{2}-e_{3}\end{array}$

From above, we see that there are two positive roots corresponding to $S U(2)$, so the particle will behave as $\mathbf{2}$ of $S U(2)$. There is only one positive root for the $S U(3)$ case, so it is a singlet for $S U(3)$. Thus, this is a $(\mathbf{2}, \mathbf{1})$ of $S U(2) \times S U(3)$ (corresponding to $H_{2}^{u}$ as in Table II). Notice that the above calculation implies that $e_{2}-e_{4}$ yields the same particle. Next, let us try another positive root, say $-e_{0}+e_{2}+e_{3}+e_{5}$. The curve equation is $f_{2}+f_{3}+f_{5}=a-b-c-d+Y=0$. Then, we get

$S U(2)$

$S U(3)$
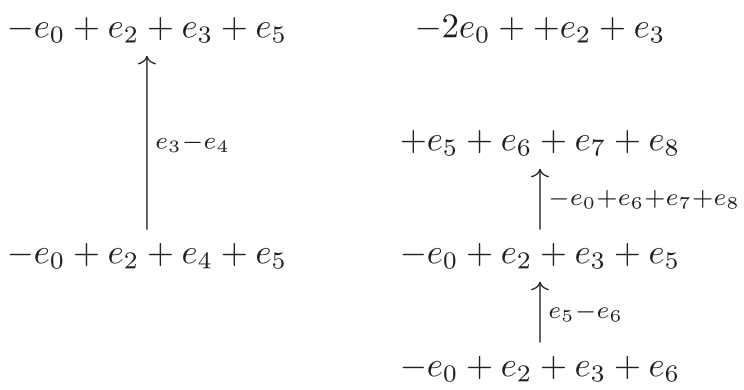

So by counting the positive roots, we conclude that it is $\mathbf{2}$ for $S U(2)$ and $\mathbf{3}$ or $\overline{\mathbf{3}}$ for $S U(3)$. As fundamental and antifundamental are just a convention, we call this order of adding $e_{5}-e_{6}$ and $-e_{0}+e_{6}+e_{7}+e_{8}$ associated with fundamental 3. Thus this is a $(\mathbf{2}, \mathbf{3})$ of $S U(2) \times S U(3)$. Last, for completeness, we will illustrate the case of $\overline{\mathbf{3}}$ with $-e_{0}+e_{2}+e_{3}+e_{4}$. The curve equation is $f_{2}+f_{3}+f_{4}=a-b-c-d-4 Y=0$. Then, we get

$$
S U(2) \quad S U(3)
$$

No other positive root $\quad-2 e_{0}+e_{2}+e_{3}$

$$
\begin{gathered}
+e_{4}+e_{5}+e_{7}+e_{8} \\
e_{5}-e_{6} \uparrow \\
-2 e_{0}+e_{2}+e_{3}+e_{4} \\
-e_{0}+e_{2}+e_{3}+e_{4} \quad e_{6}+e_{7}+e_{8} \\
-e_{0}+e_{6}+e_{7}+e_{8} \uparrow \\
-e_{0}+e_{2}+e_{3}+e_{4}
\end{gathered}
$$

Notice that the order of adding $e_{5}-e_{6}$ and $-e_{0}+e_{6}+$ $e_{7}+e_{8}$ is reversed from the previous case, so, by the above convention, this is a $(\mathbf{1}, \overline{\mathbf{3}})$ of $S U(2) \times S U(3)$. Bourjaily et al. [11] have already worked out the breaking for us. The charges for relevant particles in this paper are presented in Table II. The location of the singularity associating with a particle is a linear combination of moduli weighted by the charges. For instance, the location of $Q_{1}$ is the curve that satisfies

$$
a+b-c-d+Y=0 .
$$

\section{YUKAWA COUPLING FROM VOLUME OF THE THREE-CYCLE}

In the superpotential, a cubic term $A B C$ is allowed at tree level if the product transforms as a singlet under the gauge group. In particular, that implies the sum of charges for each of the $U(1)$ 's is zero. If such a term happens, each of the particles $A, B$, and $C$ will live on a different conical singularity which corresponds to different points $t_{A}, t_{B}$, and $t_{C}$ on the base $W$ which are solutions of equations derived from Table II (similar to Sec. (4.6). The idea of this section is that the Yukawa coupling coefficient of this term is proportional to the exponential of the volume of the threecycle wrapping around the three singularities

$$
\text { Yukawa coupling }=n_{A B C} \frac{e^{-\operatorname{Vol}\left(\Sigma_{A B C}\right)}}{\Lambda_{A B C}},
$$

where $\Sigma_{A B C}$ is the three-cycle wrapping around the singularities, $n_{A B C}$ is the sign of the term which depends subtly 
on the orientation of the three-cycle [16], ${ }^{10}$ and $\Lambda_{A B C}$ is a scale factor which is approximately the volume of the $G_{2}$ manifold. We will temporarily ignore both of $n_{A B C}$ and $\Lambda_{A B C}$ in our analysis in this section.

We are interested in the limit where gravity decouples. The $G_{2}$ manifold here is treated as large enough to make the calculation manageable. Then, we can focus on a local patch of $M_{3}$ which is approximately $\mathbb{R}^{3}$. The volume of the three-cycle in the linearization has been roughly formulated by [11]. However, a more complete analysis shows the requirement of the harmonic condition and relative rotations of the fields. By Bogomol'nyi-Prasad-Sommerfield (BPS) equations [16], locally for each moduli $\phi(\phi=a, b$, $c, d$, and $Y$; these are the $f_{i}$ 's in the previous sections), there is a harmonic function $h_{\phi}$ on the $M_{3}$ base so that $\phi=d h_{\phi}$ [16]. For simplicity, we think of $\phi$ as a three-vector, and $\phi=\nabla h_{\phi}$. The harmonic condition requires that $\Delta h_{\phi}=0$. That means

$$
\partial_{i} \phi^{i}=0 .
$$

This requires that on a linear level,

$$
\phi=H t+v,
$$

where $H$ is a real traceless symmetric $3 \times 3$ matrix, $v$ is a real three-vector, $t$ is a local real parametrization of the $3 \mathrm{D}$ base. Then, $h_{\phi}$ will have the form

$$
\frac{1}{2} t^{T} H t+v^{T} t+c
$$

where $c$ is a constant term.

The location of a particle, say $X$, is a zero $t_{X}$ of a linear combination $\phi_{X}$ of $a, b, c, d$, and $Y$ with the charges from Table II. From previous discussion, $t_{X}$ is the critical point of a harmonic function $h_{\phi_{X}}$. Assume the critical points are isolated. This is the same as assuming $H_{X}$ is invertible. The critical point of $h_{\phi_{X}}$ or the zero point of $\phi_{X}$ is

$$
t_{X}=-H_{X}^{-1} v_{X}
$$

Then, if the $A B C$ term is allowed, i.e., $h_{\phi_{A}}+h_{\phi_{B}}+h_{\phi_{C}}=0$, the volume for the three-cycle wrapping the three critical points $t_{A}, t_{B}$, and $t_{C}$ is ${ }^{11}$

$$
\begin{aligned}
\operatorname{Vol}\left(\Sigma_{A B C}\right)= & h_{\phi_{A}}\left(t_{A}\right)+h_{\phi_{B}}\left(t_{B}\right)+h_{\phi_{C}}\left(t_{C}\right) \\
= & \frac{1}{2}\left(-v_{A}^{T} H_{A}^{-1} v_{A}-v_{B}^{T} H_{B}^{-1} v_{B}\right. \\
& \left.+\left(v_{A}+v_{B}\right)^{T}\left(H_{A}+H_{B}\right)^{-1}\left(v_{A}+v_{B}\right)\right) .
\end{aligned}
$$

\footnotetext{
${ }^{10}$ Details of how to determine $n_{A B C}$ is in [16] and Appendix F of [38].

${ }^{1}$ Reference [16] gives a formulation for the general case, which has been applied to this linear case.
}

TABLE II. Relevant particles from three families of $E_{6}$; for a complete listing see [11].

\begin{tabular}{lccccccc}
\hline \hline & $S U_{3}$ & $S U_{2}$ & $U_{1}^{a}$ & $U_{1}^{b}$ & $U_{1}^{c}$ & $U_{1}^{d}$ & $U_{1}^{Y}$ \\
\hline$Q_{1}$ & $\mathbf{3}$ & $\mathbf{2}$ & 1 & 1 & -1 & -1 & 1 \\
$Q_{2}$ & $\mathbf{3}$ & $\mathbf{2}$ & 1 & -1 & -1 & -1 & 1 \\
$Q_{3}$ & $\mathbf{3}$ & $\mathbf{2}$ & -2 & 0 & -1 & -1 & 1 \\
$u_{1}^{c}$ & $\overline{\mathbf{3}}$ & $\mathbf{1}$ & 1 & 1 & -1 & -1 & -4 \\
$u_{2}^{c}$ & $\overline{\mathbf{3}}$ & $\mathbf{1}$ & 1 & -1 & -1 & -1 & -4 \\
$u_{3}^{c}$ & $\overline{\mathbf{3}}$ & $\mathbf{1}$ & -2 & 0 & -1 & -1 & -4 \\
$d_{1}^{c}$ & $\overline{\mathbf{3}}$ & $\mathbf{1}$ & 1 & 1 & -1 & 3 & 2 \\
$d_{2}^{c}$ & $\overline{\mathbf{3}}$ & $\mathbf{1}$ & 1 & -1 & -1 & 3 & 2 \\
$d_{3}^{c}$ & $\overline{\mathbf{3}}$ & $\mathbf{1}$ & -2 & 0 & -1 & 3 & 2 \\
$L_{1}$ & $\mathbf{1}$ & $\mathbf{2}$ & 1 & 1 & -1 & 3 & -3 \\
$L_{2}$ & $\mathbf{1}$ & $\mathbf{2}$ & 1 & -1 & -1 & 3 & -3 \\
$L_{3}$ & $\mathbf{1}$ & $\mathbf{2}$ & -2 & 0 & -1 & 3 & -3 \\
$H_{1}^{u}$ & $\overline{\mathbf{1}}$ & $\mathbf{2}$ & 1 & 1 & 2 & 2 & 3 \\
$H_{2}^{u}$ & $\overline{\mathbf{1}}$ & $\mathbf{2}$ & 1 & -1 & 2 & 2 & 3 \\
$H_{3}^{u}$ & $\overline{\mathbf{1}}$ & $\mathbf{2}$ & -2 & 0 & 2 & 2 & 3 \\
$H_{1}^{d}$ & $\overline{\mathbf{1}}$ & $\mathbf{2}$ & 1 & 1 & 2 & -2 & -3 \\
$H_{2}^{d}$ & $\overline{\mathbf{1}}$ & $\mathbf{2}$ & 1 & -1 & 2 & -2 & -3 \\
$H_{3}^{d}$ & $\overline{\mathbf{1}}$ & $\mathbf{2}$ & -2 & 0 & 2 & -2 & -3 \\
$e_{1}^{c}$ & $\mathbf{1}$ & $\mathbf{1}$ & 1 & 1 & -1 & -1 & 6 \\
$e_{2}^{c}$ & $\mathbf{1}$ & $\mathbf{1}$ & 1 & -1 & -1 & -1 & 6 \\
$e_{3}^{c}$ & $\mathbf{1}$ & $\mathbf{1}$ & -2 & 0 & -1 & -1 & 6 \\
\hline \hline & & & & & & & \\
\hline \hline
\end{tabular}

Notice that the constant $c$ in Eq. (5.4) plays no role here due to cancellation, so in practice, we will simply drop it. In Sec. VIC, explicit computation for a Yukawa coupling is shown for a quark term.

\section{A. Discussion of other features}

So far, we have only considered $M_{3}$ as a flat $\mathbb{R}^{3}$ which obviously overlooks the very stringent global structure of a compact $G_{2}$ manifold. This structure may reduce the parametrization freedom we have in the flat local case. The singularities' curves may also cut each other at some point beyond the local area due to compactness, increasing the number of possible Yukawa couplings. Additionally, the sign factors in Eq. (5.1) may also change the mass matrix significantly. They are determined by the gradient flow of the $h_{\phi}[16,39,40]$. It is difficult to study the gradient flow between singular points for the local model as the space is not compact. Future study of the gradient flows and hence the sign factors can reveal more of the mass matrix. As mentioned in Sec. IV A, we should project out particles we do not plan to include in our theory. Projecting a specific particle includes requiring that the curves never satisfy the particle's equation derived from Table II. That would create more restraint on the parameters. For our local case in particular, it would require a vanishing determinant of a certain linear combination of $H_{\phi}$ 's. Nonetheless, the problems with these particles are not detrimental and can be 
remedied by other means. Careful study is needed on this issue.

\section{QUARK TERMS}

\section{A. General quark terms}

Recall that the quarks get mass when the Higgses receive VEVs. For example,

$$
\lambda^{i j} H_{k}^{u} Q_{i} u_{j} \rightarrow\left\langle H_{k}^{u}\right\rangle \lambda^{i j} Q_{i}^{k} u_{j}^{k} .
$$

Ellis et al. [41] showed that $\tan \beta \approx 7$, from electroweak symmetry breaking, so we know both up and down VEVs in the two-Higgs-doublets model. We will discuss later how to adapt these into the six Higgs doublets in this paper. Quark terms that satisfy the vanishing sum of charges are

$$
\begin{aligned}
& Q_{1} u_{2}^{c} H_{3}^{u}+Q_{2} u_{1}^{c} H_{3}^{u}+Q_{1} d_{2}^{c} H_{3}^{u}+Q_{2} u_{1}^{c} H_{3}^{u} \\
& \quad+Q_{2} u_{3}^{c} H_{1}^{u}+Q_{3} u_{2}^{c} H_{1}^{u}+Q_{2} d_{3}^{c} H_{1}^{u}+Q_{3} u_{2}^{c} H_{1}^{u} \\
& \quad+Q_{3} u_{1}^{c} H_{2}^{u}+Q_{1} u_{3}^{c} H_{2}^{u}+Q_{3} d_{1}^{c} H_{2}^{u}+Q_{1} u_{3}^{c} H_{2}^{u} .
\end{aligned}
$$

Note that there is no diagonal term in this general setting. Also, some couplings between the Higgs and the quarks which could have been possible in SM are forbidden here due to the extra $U(1)$ 's. Nonetheless, those terms can still be generated by the Giudice-Masiero mechanism after the breaking of supergravity [42,43]. However, we will leave this mechanism to future study in the context of M-theory with $E_{8}$ orbifold. In the following sections, we will focus on the simplest constraints on the moduli to make the theory physical.

The relevant terms for leptons are

$$
\begin{gathered}
L_{1} e_{2}^{c} H_{3}^{d}+L_{2} e_{3}^{c} H_{1}^{d}+L_{3} e_{1}^{c} H_{2}^{d}+ \\
L_{1} \nu_{2}^{c} H_{3}^{u}+L_{2} \nu_{3}^{c} H_{1}^{u}+L_{3} \nu_{1}^{c} H_{2}^{\nu} .
\end{gathered}
$$

Notice that we only have Dirac mass terms here. Majorana terms may require quartic level, extra particles getting a VEV, or extra constraints on the moduli, so we will not discuss such terms in this paper.

\section{B. Diagonal terms and setting $\boldsymbol{a}=\mathbf{0}$}

Equation (6.2) shows that there is no diagonal term for the quark matrices. This appears to be a problem because with the top quark mass much larger than those of up and charm quarks, the trace of the mass matrix must be nonzero. This problem is generic in our method of constructing three families from $E_{8}$ singularity. The same issue was discussed in the F-theory context in [14]. The reason for this is the conservation of charge in $a$ and $b$. Hence, this directly relates to the separation of families because $a$ and $b$ break the adjoint of $E_{8}$ into three $27^{\prime} s$ in $E_{6}$. So, particles in the same family must have the same charge in $a$ and $b$, making it impossible for them to form a singlet cubic term within the same family in a generic setting. One way to remedy this is to introduce a self-intersecting curve for the up-type when $Y=0$ [14], using the fact that in grand unified theories $u$ and $Q$ both stay on the same curve of 10 of $S U(5)$. However, this method cannot be applied for downtype as $d$ does not stay on the same curve as $Q$. Moreover, self-intersecting requires higher order than linearization which we will not pursue here. Alternatively, Bourjaily et al. [11] also discuss the contribution of quartic terms. This will require giving large VEVs for extra particles, creating more parameters which we will not consider at this time. In this paper, we can consider some constraint on $a$ and $b$ leading to possible nonzero diagonal terms. This in essence sets a relation for $a$ and $b$ charges. We still keep in mind the condition of nonvanishing volumes in Eq. (4.4) as we do not wish to unnecessarily enhance the gauge symmetry. The simplest constraint we can make is $a=0$. Although it is intriguing to study other constraints, we will ignore them in this paper. This constraint will restrict the gauge group to $S U(3) \times S U(2) \times U(1)^{Y} \times$ $U(1)^{b} \times U(1)^{c} \times U(1)^{d}$. In terms of geometry, this breaking of $U(1)^{a}$ is equivalent to restricting the basis two-cycles in a linear relation, reducing the number of independent two-cycles and hence number of $U(1)$ 's.

\section{Quark mass matrices}

After setting $a=0$ together with the localization, the uptype quark mass matrix can be computed. We will show one example of the computation here for $M_{12}^{u} u_{1} u_{2}^{c}$. It comes from the term

$$
\lambda_{123}^{u} Q_{1} u_{2}^{c} H_{3}^{u}
$$

When the Higgs gets VEV at low scale, the term becomes

$$
\lambda_{123}^{u}\left\langle H_{3}^{u}\right\rangle u_{1} u_{2}^{c},
$$

where $M_{12}^{u}=\lambda_{123}^{u}\left\langle H_{3}^{u}\right\rangle$. Then, all that is left is to compute $\lambda_{123}^{u}$. At high scale, $\lambda_{123}^{u}$ can be calculated from Eq. (5.6) and Table II. In the linearization language

$$
\begin{gathered}
H_{Q_{1}}=H_{b}-H_{d}+H_{Y}, \\
v_{Q_{1}}=v_{b}-v_{d}+v_{Y}, \\
H_{u_{2}}=-H_{b}-H_{d}-4 H_{Y}, \\
v_{u_{2}}=-v_{b}-v_{d}-4 v_{Y},
\end{gathered}
$$

and then Eq. (5.6) gives 


$$
\begin{aligned}
& \operatorname{Vol}\left\{\Sigma_{Q_{1} u_{2}^{c} H_{3}^{u}}\right\}= \\
& \frac{1}{2}\left(\left(v_{b}-v_{d}+v_{Y}\right)^{T}\left(H_{b}-H_{d}+H_{Y}\right)^{-1}\left(v_{b}-v_{d}+v_{Y}\right)+\left(-v_{b}-v_{d}-4 v_{Y}\right)^{T}\left(-H_{b}-H_{d}-4 H_{Y}\right)^{-1}\left(-v_{b}-v_{d}-4 v_{Y}\right)\right. \\
& \left.\quad+\left(2 v_{d}+3 v_{Y}\right)^{T}\left(+2 H_{d}+3 H_{Y}\right)^{-1}\left(2 v_{d}+3 v_{Y}\right)\right)
\end{aligned}
$$

Thus, Eq. (5.1), ignoring the overall scaling, gives

$$
\begin{aligned}
\lambda_{123}^{u}= & n_{12}^{u} \exp \left\{-\frac{1}{2} \mid\left(v_{b}-v_{d}+v_{Y}\right)^{T}\left(H_{b}-H_{d}+H_{Y}\right)^{-1}\left(v_{b}-v_{d}+v_{Y}\right)\right. \\
& \left.+\left(-v_{b}-v_{d}-4 v_{Y}\right)^{T}\left(-H_{b}-H_{d}-4 H_{Y}\right)^{-1}\left(-v_{b}-v_{d}-4 v_{Y}\right)+\left(2 v_{d}+3 v_{Y}\right)^{T}\left(+2 H_{d}+3 H_{Y}\right)^{-1}\left(2 v_{d}+3 v_{Y}\right) \mid\right\} .
\end{aligned}
$$

Then, we have to run these Yukawa couplings down to the SM scale to compute the mass. Note that the diagonal term $Q_{3} u_{3}^{c} H_{3}^{c}$, obtained from setting $a=0$, can be computed by the above method.

\section{Six Higgs VEVs}

In the six Higgs doublets model without extra $U(1)$ 's, one can choose a basis for up-type and down-type Higgses so that only one pair of Higgses gets a VEV without loss of generality. Here, due to different charges for the Higgses from the extra $U(1)$ 's (see Table II), we cannot make such a choice of basis.

We will try to translate from the two VEVs of SM Higgses to the six VEVs in our theory. By standard QFT, we can relate this by looking at the mass of the $W$ boson in the SM and identify

$$
\begin{aligned}
& \left\langle H_{u}^{\mathrm{SM}}\right\rangle^{2}=\sum_{i}\left\langle H_{u}^{i}\right\rangle^{2}, \\
& \left\langle H_{d}^{\mathrm{SM}}\right\rangle^{2}=\sum_{i}\left\langle H_{d}^{i}\right\rangle^{2} .
\end{aligned}
$$

So, we can use spherical parametrization to write

$$
\begin{aligned}
\left\langle H_{u / d}^{1}\right\rangle & =\left\langle H_{u / d}^{\mathrm{SM}}\right\rangle \cos \phi_{u / d} \sin \theta_{u / d}, \\
\left\langle H_{u / d}^{2}\right\rangle & =\left\langle H_{u / d}^{\mathrm{SM}}\right\rangle \sin \phi_{u / d} \sin \theta_{u / d}, \\
\left\langle H_{u / d}^{3}\right\rangle & =\left\langle H_{u / d}^{\mathrm{SM}}\right\rangle \cos \theta_{u / d} .
\end{aligned}
$$

Such Higgs VEVs can lead to flavor changing neutral currents (FCNC). We keep the mixing angles small and assume no problems with FCNC, which implies $\theta \ll 1$.

\section{E. Toward physical coupling}

Note that the Yukawa couplings in M-theory belong to the high energy scale. We will attempt to use the already existent list of high scale Yukawa coupling running from
SM experimental Yukawas in Table 1 of [44] ${ }^{12}$ and find a solution for our parameters. We assume the effect of the extra U(1)'s from our theory in the renormalization group equations (RGEs) is not significant, and the Yukawas have approximately the same magnitudes as in [44].

In order to compare with physical Yukawa couplings, we need to take into account a few modifications. First, as mentioned in [14], we need a scaling factor to normalize the wave function. For cubic Yukawa, it is roughly proportional to $V_{G_{2}}^{-\frac{1}{2}}$ where $V_{G_{2}}$ is the volume of the $G_{2}$ manifold and still a parameter in our theory (as a local model cannot determine the global volume). Thus the scaling factor for all the cubic Yukawas is a parameter in this local model.

\section{F. Higgs VEVs}

On the other hand, recall that the Higgses only get VEVs at low scale. Therefore, precisely speaking, we can only consider the VEVs of the six Higgses after we run our M-theory Yukawa couplings down to low scale. Unfortunately, at high scale, we only have a set of algebraic expressions for M-theory Yukawas, making the running down to low scale complicated. Moreover, we cannot directly fit our Yukawas with the existing data of high scale running from SM Yukawas because they all assume a two Higgses model. Therefore, to remedy this problem, we will use a heuristic treatment assuming that the angular factors, in Eqs. (6.16), are regarded as part of the low scale Yukawa couplings and do not change much while running to high scale. Then, the effective VEVs at low scale are just the two VEVs from the SM, and the Yukawa couplings at high scale used to fit with Table 1 of [44] then are

$$
Y=f(\phi, \theta) \lambda,
$$

\footnotetext{
${ }^{12}$ The grand unified theory (GUT) group is slightly different, but we assume the magnitude of the couplings are approximately the same. See also [45].
} 
where $\lambda$ is a Yukawa computed from Sec. VIC and $f(\phi, \theta)$ is one of the angular functions associated with the Higgs fields from Eqs. (6.16). The full tables of high scale Yukawa couplings with angular factors are presented in Tables IV-VI Appendix.

\section{YUKAWA MATRIX FOR GAUGE GROUP $S U(3) \times S U(2) \times U(1)^{Y} \times U(1)^{b} \times U(1)^{c} \times U(1)^{d}$}

First, we need to fix all extra degrees of freedom. Translation allows setting $v_{d}=0$. We also have 3 degrees of rotation and 1 degree of scaling to make $v_{b}=(1,0,0)$.

Second, we will try to consider the scattering around special cases of $H_{b}$ and $H_{d}$. Notice from the list in Eq. (4.4) that by setting all parameters to zero except $b$, we see that volumes of root $e_{1}-e_{2}$ and $e_{2}-e_{3}$ are controlled by $b$. They are responsible for breaking the adjoint of $E_{8}$ into three 27's of $E_{6}$ (see Fig. 3), and hence are also responsible for separating the three SM families.

On the other hand, $d$ controls $e_{2}-e_{3}, e_{6}-e_{7}$, and $e_{7}-e_{8}$. The blown-up two-cycle of $e_{2}-e_{3}$ breaks the adjoint of $E_{8}$ into two 27's of $E_{6}$, which transform as the fundamental and singlet of $S U(2)$, respectively, i.e., $(\mathbf{2 7}, \mathbf{2}) \oplus(\mathbf{2 7}, \mathbf{1})$. Thus $d$ separates one family (the top quark family) from the other two in the adjoint of $E_{8}$. The latter still has an $S U(2)$ family symmetry (which is broken when we turn $b$ on). Additionally, $e_{6}-e_{7}$ corresponds to breaking the 27's of $E_{6}$ into the presentations of $S O(10)$, separating the Higgses from quarks and leptons. Finally, $e_{7}-e_{8}$ splits the 16's of $S O(10)$ into the 10 and $\overline{\mathbf{5}}$ of $S U(5)$. Thus, $d$ also separates the up-type quarks (up, charm, top) from the down-type quarks (down, strange, bottom), i.e., an isospin breaking effect.

\section{NUMERICAL EVALUATION}

To test the compatibility of this model with the Standard Model, we perform a regression on the free parameters by a least squares approach. Our calculations of Yukawa couplings are compared to experimentally measured weak scale Yukawa couplings which have been run up to the GUT scale. ${ }^{13}$ The theoretical uncertainty in the calculation dominates over the experimental uncertainties, and we only consider theoretical uncertainty when minimizing the sum of the residuals.

Using previous arguments, we set the base parameters corresponding to $a=0$ to zero, $v_{d}$ to zero, and $v_{b}$ to $(1,0$, $0)$. With three $3 \times 3$ traceless symmetric matrices $H_{\phi}$ and two 3-vectors, we have 18 free parameters from the base space. We have four additional parameters from the Higgs VEVs, satisfying $\left\langle\left(H_{1}^{2}+H_{2}^{2}+H_{3}^{2}\right)^{1 / 2}\right\rangle=\left\langle H_{\text {MSSM }}\right\rangle$. Although we have more free parameters than constraints from the data, the nonlinearity in calculating the Yukawas restricts the solutions. A list of numerical solutions is in

\footnotetext{
${ }^{13}$ See also [45].
}
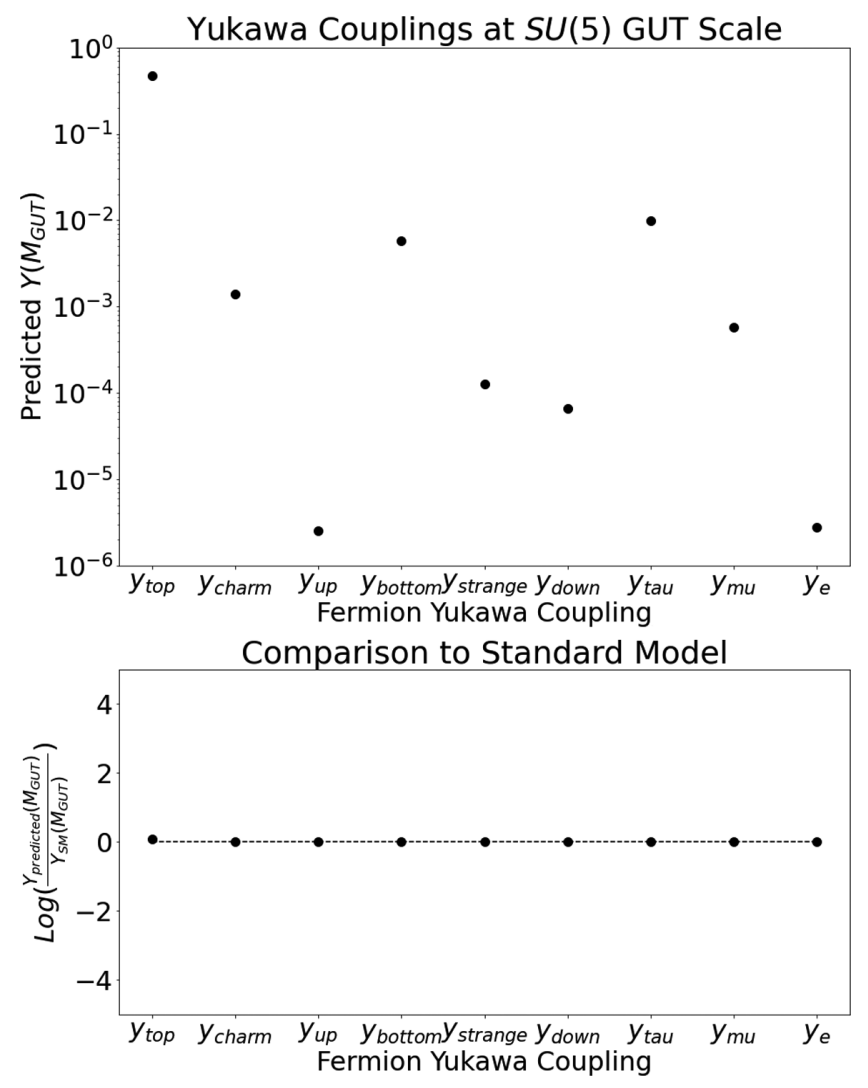

FIG. 4. A set of sample solutions found numerically. The three symbols indicate three different solutions, and the line indicates the measured value for each Yukawa coupling.

Appendix A. A set of samples from numerical evaluation is shown in Fig. 4. We have observed some general trends among the numerical solutions. Most importantly, there exists a hierarchy of Yukawas within each family which come from the breaking of the flavor and family symmetries. There is a large top quark Yukawa coupling. Finally, it appears that the hierarchy solution only happens when $\theta$ is small, an observation that is expected from the aforementioned no-neutral-current condition.

\section{EFFECT OF THE HIGGSES AND YUKAWA COUPLINGS}

We want to use this section to emphasize the necessity of both the Higgs sector and the Yukawa exponential factor (which is of stringy origin) in satisfying the hierarchy. First, if only one family of the Higgses get VEVs, say $\mathrm{H}_{3}$, we will get the up-type quark matrix of the form

$$
\left\{\begin{array}{lll}
0 & A & 0 \\
A & 0 & 0 \\
0 & 0 & B
\end{array}\right\} .
$$

Although we still have a hierarchy with one heavy and two light families. There is no hierarchy between the lighter two. 
Second, if all three Higgs families get VEVs while all the Yukawa coefficients are the same (equal to 1), the theory will not have the physical hierarchy. Considering only the angular factors (dropping the common VEV factor), we have the matrix in the form

$$
\left\{\begin{array}{ccc}
0 & \tilde{A} & \tilde{B} \\
\tilde{A} & 0 & \tilde{C} \\
\tilde{B} & \tilde{C} & \tilde{A}
\end{array}\right\} .
$$

Then, from the characteristic equation, we conclude

$$
\left\{\begin{array}{l}
\lambda_{1}+\lambda_{2}+\lambda_{3}=\tilde{A}, \\
\lambda_{1} \lambda_{2}+\lambda_{2} \lambda_{3}+\lambda_{3} \lambda_{1}=\tilde{A}^{2}+\tilde{B}^{2}+\tilde{C}^{2}=1
\end{array}\right.
$$

This can be shown to imply that the quark hierarchy can never satisfy equations (9.1). Therefore, both the three families of Higgses and the stringy Yukawa suppression are needed for the hierarchy.

\section{ANOMALIES}

The theory may result in gauge boson triangle anomalies. Such an anomaly can be canceled by the StückelbergGreen-Schwarz mechanism and gives some bosons a mass.

\section{A. How to compute the anomaly}

We focus our attention on a model with gauge group $S U(3) \times S U(2) \times U(1)^{n}$ where the $U(1)$ 's are to be examined. It can be shown that anomalies of the $U(1)$ 's come from a triangle loop of bosons in three configurations: $S U(3)-S U(3)-U(1)$ and $S U(2)-S U(2)-U(1)$ and $U(1)-U(1)-U(1)$. The anomaly of a triangle from three $U(1)$ 's is proportional to the sum of particles that transform under the non-Abelian factor weighted by the charge of $U(1)$ factors. If this sum is zero, the configuration of $U(1)$ 's is anomaly-free. Otherwise, it is anomalous.

Explicitly, for

(i) $U^{a}(1)-U^{b}(1)-U^{c}(1)$ is simply the sum, over all the particles, of the products of $\mathrm{U}(1)$ charges: $\sum_{i: \text { all particles }} q_{i}^{a} q_{i}^{b} q_{i}^{c}$. (ii) $S U(3)-S U(3)-U(1)$ : Sum of U(1) charges over all triplets: $\sum_{i}$ all triplets $q_{i}$.

(iii) $S U(2)-S U(2)-U(1)$ : Sum of U(1) charges over all doublets: $\sum_{i}$ all doublets $q_{i}$.

Note that $(3,2)$ has three $S U(2)$ doublets and two $S U(3)$ triplets.

\section{B. Anomaly cancellation by Stückelberg-Green- Schwarz mechanism}

In string theory, an additional term is added to cancel out the anomaly [46]. Such a term will give a mass to the anomalous boson. This is called the StückelbergGreen-Schwarz mechanism. The anomaly-related terms in effective action is

$$
\begin{aligned}
\mathcal{S}= & -\sum_{i} \int d^{4} x \frac{1}{4 g_{i}^{2}} F_{i, \mu \nu} F_{i}^{\mu \nu} \\
& -\underbrace{\frac{1}{2} \int d^{4} x \sum_{I}\left(\partial_{\mu} a^{I}+M_{i}^{I} A_{\mu}^{i}\right)^{2}}_{\text {Stückelberg term }} \\
& +\underbrace{\frac{1}{24 \pi^{2}} C_{i j}^{I} \int a^{I} F^{i} \wedge F^{j}}_{\text {Green-Schwarz term }} \\
& +\underbrace{\frac{1}{24 \pi^{2}} E_{i j, k} \int A^{i} \wedge A^{j} \wedge F^{k}}_{\text {Chern-Simons term }},
\end{aligned}
$$

where $a^{I}$ are axions, $C_{i j}^{I}$ is symmetric, $E_{i j k}$ is symmetric between $i$ and $j$. Then, when the anomalous variation is distributed democratically among the three vertices, the condition for canceling the anomalies is

$$
t_{i j k}+E_{i j k}+E_{i k j}+M_{i}^{I} C_{j k}^{I}=0,
$$

where $t_{i j k}=\operatorname{Tr}\left\{t_{i} t_{j} t_{k}\right\}$. We now focus on the anomalies coming from the $U(1)-U(1)-U(1)$ triangle which are computed in Table III. Then, the generators are commuting, so $t_{i j k}$ is totally symmetric. Summing all equations of permutation of $i, j$, and $k$, we get

TABLE III. Anomaly computation.

\begin{tabular}{lccccccc}
\hline \hline$a-a-a$ & -78 & $b-b-b$ & -6 & $c-c-c$ & 96 & $d-d-d$ & 0 \\
$Y-Y-Y$ & 0 & $a-a-b$ & 18 & $a-a-c$ & -96 & $a-a-d$ & 0 \\
$a-a-Y$ & 0 & $b-b-a$ & 26 & $b-b-c$ & -32 & $b-b-d$ & 0 \\
$b-b-Y$ & 0 & $c-c-a$ & 0 & $c-c-b$ & 0 & $c-c-d$ & 0 \\
$c-c-Y$ & 0 & $d-d-a$ & 0 & $d-d-b$ & 0 & $d-d-c$ & -288 \\
$d-d-Y$ & 0 & $Y-Y-a$ & 0 & $Y-Y-b$ & 0 & $Y-Y-c$ & -288 \\
$Y-Y-d$ & 0 & $a-b-c$ & 0 & $a-b-d$ & 0 & $a-b-Y$ & 0 \\
$a-c-d$ & 0 & $a-c-Y$ & 0 & $a-d-Y$ & 0 & $b-c-d$ & 0 \\
$b-c-Y$ & 0 & $b-d-Y$ & 0 & $c-d-Y$ & 288 & $\ldots$ & $\ldots$ \\
\hline \hline
\end{tabular}




$$
M_{i}^{I} C_{j k}^{I}+M_{j}^{I} C_{k i}^{I}+M_{k}^{I} C_{i j}^{I}=-3 t_{i j k}
$$

where we used $E_{i j k}=-E_{j i k}$. We can use the value of $t_{i j k}$ to compute possible values for $M_{i}^{I}$ and $C_{i j}^{I}$.

Notice that simultaneous transformations

$$
M_{i}^{I} \rightarrow a^{I} M_{i}^{I}, \quad C_{i j}^{I} \rightarrow \frac{1}{a^{I}} C_{i j}^{I}
$$

for all $i, j$ leaves the equations invariant. So, if (10.4) has a solution, the solution will only be unique up to the ratio of the masses. For the anomaly of $b-b-b$, the system is simply reduced to one linear equation giving $U(1)^{b}$ a nonzero mass, up to a scaling,

$$
M_{b}=-3 t_{b b b}=18 \text {. }
$$

This specific number does not mean much due to scaling freedom. ${ }^{14}$ The only significant point is $U(1)^{b}$ being massive. Similarly, $U(1)^{c}$ is also massive. Unfortunately, $U(1)^{d}$ is anomaly-free and hence cannot get mass this way. Yet, as the Higgses are charged in $U(1)^{d}\left[U(1)^{b}\right.$ as well], their electroweak VEVs can give mass to the bosons.

\section{STABILIZATION}

One would naturally ask if the solution we found is indeed a solution that stabilizes the $G_{2}$ manifold. Our argument is that our local moduli solution can be stabilized by appropriate global moduli. Notice that in our local theory, the local moduli control the resolution of $E_{8}$ singularity but are not a complete set of moduli controlling the global $G_{2}$ manifold. Acharya et al. [3] showed that M-theory is stabilized on a large class of smooth $G_{2}$ manifolds. Such a smooth formulation does not precisely describe the singular manifold in our model, but we assume that our local singular region is small enough so that the contribution to over all stabilization will be a perturbation from the equations from arXiv:hep-th/0701034. After all, the linear formulation for the $a, b, c, d, Y$ in our paper requires locality; otherwise, linear order is not enough to describe the theory.

Moreover, we can see that heuristically, the moduli is stabilizable from the formulation of Acharya et al. through the remaining global moduli that do not control the singularities. Recall that $a, b, c, d, Y$ control the volume of the two-cycles resolving the $E_{8}$ singularity. These cycles intersect transversely according to the Dynkin diagram. Two intersecting two-cycles will make a complex two torus. Thus, roughly the volume of manifold is locally

\footnotetext{
${ }^{14}$ Study of anomaly involving $S U(2)$ and $S U(3)$ may fix this freedom.
}

$V \sim \sum r_{1} \times r_{2} \times r_{3} \times\left(r_{a} \times r_{b}+r_{b} \times r_{c}+r_{c} \times r_{d}+\cdots\right)$,

where $r_{1,2,3}$ control the volume of the $M_{3}$ base, $r_{a, b, c, d, Y}$ control the volumes of the complex two tori. Then, each individual term is at the standard product form in Eq. (2) of [3]. This volume can be stabilized by exactly the same mechanism as in [3] because the equations for each $r_{i}$ agree among the terms [see Eqs. (18) and (19) of [3] ]. Notice that our model does not determine $r_{1,2,3}$, and thus their values can be stabilized accordingly to accompany $r_{a, b, c, d, Y}$ so that the stabilization equations [Eqs. (18) and (19)] are satisfied. This is only heuristic because there can be more moduli when we go global on the $G_{2}$ manifold, and there can be the coefficients for each term (constant coefficients do not effect our argument here).

This also addresses the concern of the gravity selfreaction. The effect of self-reaction on the masses can be limited by the moduli stabilization. The only way for the self-reaction to affect the masses in our model is changing the location of the singularities on the internal $G_{2}$ manifold. That will result in a change in the moduli. If the moduli are at the stable point, the self-reaction will push them out of the equilibrium. The stabilizing mechanism will kick in and restore the moduli to the stable point, canceling the effect of self-correction.

One can also refer additionally to Acharya and Bobkov [47] where they argue that the moduli stabilization mechanism produces vacua within the regime of validity of supergravity. For large Yukawas such as the top, Acharya and Witten suggest that the duality with the heterotic string suggests that there is no significant backreaction [48].

\section{CONCLUSION}

In this paper, we use the geometric gauge breaking mechanism in M-theory compactified on a singular $G_{2}$ manifold to help understand quark and charged lepton masses. We start with the adjoint representation of a single $E_{8}$ that contains exactly three related families of quarks and leptons. Then, we break $E_{8}$ to the Standard Model via deformations and geometric engineering, following the technique of Katz and Morrison [28]. We explicitly computed Yukawa couplings in a local model and show their fitting with experimental results.

With this approach, we hope to understand the origin of flavors and three families, and the values of quark and lepton masses. We are partially successful. We can see three families and the hierarchy of quark and lepton masses emerge. We can see the isospin breaking that makes the $S U(2)$ doublets such as top and bottom, up and down, electron and electron neutrino which all have different masses and the hierarchy of family masses. The amounts are controlled by deformation parameters that are effectively moduli. We can calculate the values of 
the deformation moduli that lead to the hierarchy and realistic values for the masses. Ideally, we would be able to predict the values at which the deformation moduli are stabilized, and predict the masses, but we are not yet able to do so. In principal, the moduli have to satisfy stabilization constraints, neutrino sector, global $G_{2}$ structure, and so on. So, future study on these constraints applying to our quark and lepton context may make the theory predictive.

We are able to get some important mass values. We work with high scale Yukawa couplings. The top quark has a Yukawa coupling of order one. The up quark can be less than the down quark. More precisely, $m_{\text {up }}+m_{e} \lesssim$ $m_{\text {down }}$ (ignoring an electromagnetic contribution), so that protons will be stable rather than neutrons, allowing hydrogen atoms. We can derive the conditions in the underlying theory for this inequality, or for the top Yukawa to be of order unity, but we cannot yet show they must uniquely hold. Three families and a hierarchy of masses do arise generically. The theory might not have allowed these results, so we view obtaining them in a UV complete theory as significant progress. We do not at this stage have much control over what masses are associated with the three extra $U(1)$ 's, but none should be massless. Then the spectrum should contain four new $Z$ states.
They are well motivated. In future work it may be possible to constrain their masses. Last, we also leave the study of the remaining particles resulting from $E_{8}$ breaking for future study.

\section{ACKNOWLEDGMENTS}

We acknowledge the support from the LCTP at the University of Michigan and DoE Grant No. DESC0007859. We also thank all of Khoa's friends who helped edit the manuscript.

\section{APPENDIX: YUKAWA TABLES}

Here, $n_{i j}$ takes value $1,-1$, or 0 depending on the trivalent gradient flow existence and orientation whose details are in [16]. We will assume they are all 1 in this local model. $H$ and $v$ explicitly are

$$
H_{\phi}=\left\{\begin{array}{ccc}
u_{\phi}^{1} & u_{\phi}^{3} & u_{\phi}^{4} \\
u_{\phi}^{3} & u_{\phi}^{2} & u_{\phi}^{5} \\
u_{\phi}^{4} & u_{\phi}^{5} & -u_{\phi}^{1}-u_{\phi}^{2}
\end{array}\right\}, \quad v_{\phi}=\left\{\begin{array}{c}
v_{\phi}^{1} \\
v_{\phi}^{2} \\
v_{\phi}^{3}
\end{array}\right\} .
$$

The numerical result for the moduli used in Fig. 4 is in Table VII.

TABLE IV. Up-type quark terms.

\begin{tabular}{|c|c|}
\hline $\begin{array}{l}\text { Term } \\
Q_{i} u_{j}^{c} H_{k}^{u} \\
\end{array}$ & Coupling $Y_{i j k}^{u}$ \\
\hline$Q_{1} u_{2}^{c} H_{3}^{u}$ & $\begin{array}{l}n_{12}^{u} \cos \theta_{u} \exp \left\{-\frac{1}{2}\left|\left(v_{b}-v_{c}-v_{d}+v_{Y}\right)^{T}\left(H_{b}-H_{c}-H_{d}+H_{Y}\right)^{-1}\left(v_{b}-v_{c}-v_{d}+v_{Y}\right)-\frac{1}{2}\right|\left(v_{b}-v_{c}-v_{d}+v_{Y}\right)^{T}\left(H_{b}-\right.\right. \\
\left.\quad H_{c}-H_{d}+H_{Y}\right)^{-1}\left(v_{b}-v_{c}-v_{d}+v_{Y}\right)+\left(-v_{b}-v_{c}-v_{d}-4 v_{Y}\right)^{T}\left(-H_{b}-H_{c}-H_{d}-4 H_{Y}\right)^{-1}\left(-v_{b}-v_{c}-v_{d}-\right. \\
\left.\left.\quad 4 v_{Y}\right)+\left(2 v_{d}+3 v_{Y}\right)^{T}\left(2 H_{c}+2 H_{d}+3 H_{Y}\right)^{-1}\left(2 v_{d}+3 v_{Y}\right) \mid\right\}\end{array}$ \\
\hline$Q_{1} u_{3}^{c} H_{2}^{u}$ & $\begin{array}{l}n_{13}^{u} \sin \phi_{u} \sin \theta_{u} \exp \left\{-\frac{1}{2} \mid\left(v_{b}-v_{c}-v_{d}+v_{Y}\right)^{T}\left(H_{b}-H_{c}-H_{d}+H_{Y}\right)^{-1}\left(v_{b}-v_{c}-v_{d}+v_{Y}\right)+\left(-v_{c}-v_{d}-\right.\right. \\
\left.\quad 4 v_{Y}\right)^{T}\left(-H_{c}-H_{d}-4 H_{Y}\right)^{-1}\left(-v_{c}-v_{d}-4 v_{Y}\right)+\left(-v_{b}+2 v_{d}+3 v_{Y}\right)^{T}\left(-H_{b}+2 H_{c}+2 H_{d}+3 H_{Y}\right)^{-1}\left(-v_{b}+2 v_{d}+\right. \\
\left.\left.\quad 3 v_{Y}\right) \mid\right\}\end{array}$ \\
\hline$Q_{2} u_{1}^{c} H_{3}^{u}$ & $\begin{array}{l}n_{21}^{u} \cos \theta_{u} \exp \left\{-\frac{1}{2} \mid\left(-v_{b}-v_{c}-v_{d}+v_{Y}\right)^{T}\left(-H_{b}-H_{c}-H_{d}+H_{Y}\right)^{-1}\left(-v_{b}-v_{c}-v_{d}+v_{Y}\right)+\left(v_{b}-v_{c}-v_{d}-\right.\right. \\
\left.\left.\quad 4 v_{Y}\right)^{T}\left(H_{b}-H_{c}-H_{d}-4 H_{Y}\right)^{-1}\left(v_{b}-v_{c}-v_{d}-4 v_{Y}\right)+\left(2 v_{d}+3 v_{Y}\right)^{T}\left(2 H_{c}+2 H_{d}+3 H_{Y}\right)^{-1}\left(2 v_{d}+3 v_{Y}\right) \mid\right\}\end{array}$ \\
\hline$Q_{2} u_{3}^{c} H_{1}^{u}$ & $\begin{array}{l}n_{23}^{u} \cos \phi_{u} \sin \theta_{u} \exp \left\{-\frac{1}{2} \mid\left(-v_{b}-v_{c}-v_{d}+v_{Y}\right)^{T}\left(-H_{b}-H_{c}-H_{d}+H_{Y}\right)^{-1}\left(-v_{b}-v_{c}-v_{d}+v_{Y}\right)+\left(-v_{c}-v_{d}-\right.\right. \\
\left.\quad 4 v_{Y}\right)^{T}\left(-H_{c}-H_{d}-4 H_{Y}\right)^{-1}\left(-v_{c}-v_{d}-4 v_{Y}\right)+\left(v_{b}+2 v_{c}+2 v_{d}+3 v_{Y}\right)^{T}\left(H_{b}+2 H_{c}+2 H_{d}+3 H_{Y}\right)^{-1}\left(v_{b}+2 v_{c}+\right. \\
\left.\left.\quad 2 v_{d}+3 v_{Y}\right) \mid\right\}\end{array}$ \\
\hline$Q_{3} u_{1}^{c} H_{2}^{u}$ & $\begin{array}{l}n_{31}^{u} \sin \phi_{u} \sin \theta_{u} \exp \left\{-\frac{1}{2} \mid\left(-v_{c}-v_{d}+v_{Y}\right)^{T}\left(-H_{c}-H_{d}+H_{Y}\right)^{-1}\left(-v_{c}-v_{d}+v_{Y}\right)+\left(v_{b}-v_{c}-v_{d}-4 v_{Y}\right)^{T}\left(H_{b}-H_{c}-\right.\right. \\
\left.\quad H_{d}-4 H_{Y}\right)^{-1}\left(v_{b}-v_{c}-v_{d}-4 v_{Y}\right)+\left(-v_{b}+2 v_{c}+2 v_{d}+3 v_{Y}\right)^{T}\left(-H_{b}+2 H_{c}+2 H_{d}+3 H_{Y}\right)^{-1}\left(-v_{b} 2 v_{c}+2 v_{d}+\right. \\
\left.\left.\quad 3 v_{Y}\right) \mid\right\}\end{array}$ \\
\hline$Q_{3} u_{2}^{c} H_{1}^{u}$ & $\begin{array}{l}n_{23}^{u} \cos \phi_{u} \sin \theta_{u} \exp \left\{-\frac{1}{2} \mid\left(-v_{c}-v_{d}+v_{Y}\right)^{T}\left(-H_{c}-H_{d}+H_{Y}\right)^{-1}\left(-v_{c}-v_{d}+v_{Y}\right)+\left(-v_{b}-v_{c}-v_{d}-4 v_{Y}\right)^{T}\left(-H_{b}-\right.\right. \\
\left.\quad H_{c}-H_{d}-4 H_{Y}\right)^{-1}\left(-v_{b}-v_{c}-v_{d}-4 v_{Y}\right)+\left(v_{b}+2 v_{c}+2 v_{d}+3 v_{Y}\right)^{T}\left(H_{b}+2 H_{c}+2 H_{d}+3 H_{Y}\right)^{-1}\left(v_{b}+2 v_{c}+\right. \\
\left.\left.\quad 2 v_{d}+3 v_{Y}\right) \mid\right\}\end{array}$ \\
\hline$Q_{3} u_{3}^{c} H_{3}^{u}$ & $\begin{array}{l}n_{33}^{u} \cos \theta_{u} \exp \left\{-\frac{1}{2} \mid\left(-v_{c}-v_{d}+v_{Y}\right)^{T}\left(-H_{c}-H_{d}+H_{Y}\right)^{-1}\left(-v_{c}-v_{d}+v_{Y}\right)+\left(-v_{c}-v_{d}-4 v_{Y}\right)^{T}\left(-H_{c}-H_{d}-\right.\right. \\
\left.\left.\quad 4 H_{Y}\right)^{-1}\left(-v_{c}-v_{d}-4 v_{Y}\right)+\left(2 v_{c}+2 v_{d}+3 v_{Y}\right)^{T}\left(2 H_{c}+2 H_{d}+3 H_{Y}\right)^{-1}\left(2 v_{c}+2 v_{d}+3 v_{Y}\right) \mid\right\}\end{array}$ \\
\hline All else & 0 \\
\hline
\end{tabular}


TABLE V. Down-type quark terms.

\begin{tabular}{|c|c|}
\hline $\begin{array}{l}\text { Term } \\
Q_{i} d_{j}^{c} H_{k}^{d}\end{array}$ & Coupling $Y_{i j k}^{d}$ \\
\hline$Q_{1} d_{2}^{c} H_{3}^{d}$ & $\begin{array}{l}n_{12}^{d} \cos \theta_{d} \exp \left\{-\frac{1}{2} \mid\left(v_{b}-v_{c}-v_{d}+v_{Y}\right)^{T}\left(H_{b}-H_{c}-H_{d}+H_{Y}\right)^{-1}\left(v_{b}-v_{c}-v_{d}+v_{Y}\right)+\left(-v_{b}-v_{c}+3 v_{d}+\right.\right. \\
\left.\quad 2 v_{Y}\right)^{T}\left(-H_{b}-H_{c}+3 H_{d}+2 H_{Y}\right)^{-1}\left(-v_{b}-v_{c}+3 v_{d}+2 v_{Y}\right)+\left(2 v_{c}-2 v_{d}-3 v_{Y}\right)^{T}\left(2 H_{c}-2 H_{d}-3 H_{Y}\right)^{-1}\left(2 v_{c}-2 v_{d}-\right. \\
\left.\left.\quad 3 v_{Y}\right) \mid\right\}\end{array}$ \\
\hline$Q_{1} d_{3}^{c} H_{2}^{d}$ & $\begin{array}{l}n_{13}^{d} \sin ?_{d} \sin \theta_{d} \exp \left\{-\frac{1}{2} \mid\left(v_{b}-v_{c}-v_{d}+v_{Y}\right)^{T}\left(H_{b}-H_{c}-H_{d}+H_{Y}\right)^{-1}\left(v_{b}-v_{c}-v_{d}+v_{Y}\right)+\left(-v_{c}+3 v_{d}+\right.\right. \\
\left.\quad 2 v_{Y}\right)^{T}\left(-H_{c}+3 H_{d}+2 H_{Y}\right)^{-1}\left(-v_{c}+3 v_{d}+2 v_{Y}\right)+\left(2 v_{c}-v_{b}-2 v_{d}-3 v_{Y}\right)^{T}\left(-H_{b}+2 H_{c}-2 H_{d}-3 H_{Y}\right)^{-1}\left(2 v_{c}-v_{b}-\right. \\
\left.\left.\quad 2 v_{d}-3 v_{Y}\right) \mid\right\}\end{array}$ \\
\hline$Q_{2} d_{1}^{c} H_{3}^{d}$ & $\begin{array}{l}n_{21}^{d} \cos \theta_{d} \exp \left\{-\frac{1}{2} \mid\left(-v_{b}-v_{c}-v_{d}+v_{Y}\right)^{T}\left(-H_{b}-H_{c}-H_{d}+H_{Y}\right)^{-1}\left(-v_{b}-v_{c}-v_{d}+v_{Y}\right)+\left(v_{b}-v_{c}+3 v_{d}+\right.\right. \\
\left.\quad 2 v_{Y}\right)^{T}\left(H_{b}-H_{c}+3 H_{d}+2 H_{Y}\right)^{-1}\left(v_{b}-v_{c}+3 v_{d}+2 v_{Y}\right)+\left(2 v_{c}-2 v_{d}-3 v_{Y}\right)^{T}\left(2 H_{c}-2 H_{d}-3 H_{Y}\right)^{-1}\left(2 v_{c}-2 v_{d}-\right. \\
\left.\left.\quad 3 v_{Y}\right) \mid\right\}\end{array}$ \\
\hline$Q_{2} d_{3}^{c} H_{1}^{d}$ & $\begin{array}{l}n_{23}^{d} \cos ?_{d} \sin \theta_{d} \exp \left\{-\frac{1}{2} \mid\left(-v_{b}-v_{c}-v_{d}+v_{Y}\right)^{T}\left(-H_{b}-H_{c}-H_{d}+H_{Y}\right)^{-1}\left(-v_{b}-v_{c}-v_{d}+v_{Y}\right)+\left(-v_{c}+3 v_{d}+\right.\right. \\
\left.\quad 2 v_{Y}\right)^{T}\left(-H_{c}+3 H_{d}+2 H_{Y}\right)^{-1}\left(-v_{c}+3 v_{d}+2 v_{Y}\right)+\left(v_{b}+2 v_{c}-2 v_{d}-3 v_{Y}\right)^{T}\left(H_{b}+2 H_{c}-2 H_{d}-3 H_{Y}\right)^{-1}\left(v_{b}+2 v_{c}-\right. \\
\left.\left.2 v_{d}-3 v_{Y}\right) \mid\right\}\end{array}$ \\
\hline$Q_{3} d_{1}^{c} H_{2}^{d}$ & $\begin{array}{l}n_{31}^{d} \sin ?_{d} \sin \theta_{d} \exp \left\{-\frac{1}{2} \mid\left(-v_{c}-v_{d}+v_{Y}\right)^{T}\left(-H_{c}-H_{d}+H_{Y}\right)^{-1}\left(-v_{c}-v_{d}+v_{Y}\right)+\left(v_{b}-v_{c}+3 v_{d}+2 v_{Y}\right)^{T}\left(H_{b}-H_{c}+\right.\right. \\
\left.\quad 3 H_{d}+2 H_{Y}\right)^{-1}\left(v_{b}-v_{c}+3 v_{d}+2 v_{Y}\right)+\left(-v_{b}+2 v_{c}-2 v_{d}-3 v_{Y}\right)^{T}\left(-H_{b}+2 H_{c}-2 H_{d}-3 H_{Y}\right)^{-1}\left(-v_{b}+2 v_{c}-2 v_{d}-\right. \\
\left.\left.\quad 3 v_{Y}\right) \mid\right\}\end{array}$ \\
\hline$Q_{3} d_{2}^{c} H_{1}^{d}$ & $\begin{array}{l}n_{32}^{d} \cos ?_{d} \sin \theta_{d} \exp \left\{-\frac{1}{2} \mid\left(-v_{c}-v_{d}+v_{Y}\right)^{T}\left(-H_{c}-H_{d}+H_{Y}\right)^{-1}\left(-v_{c}-v_{d}+v_{Y}\right)+\left(-v_{b}-v_{c}+3 v_{d}+2 v_{Y}\right)^{T}\left(-H_{b}-\right.\right. \\
\left.\quad H_{c}+3 H_{d}+2 H_{Y}\right)^{-1}\left(-v_{b}-v_{c}+3 v_{d}+2 v_{Y}\right)+\left(v_{b}+2 v_{c}-2 v_{d}-3 v_{Y}\right)^{T}\left(H_{b}+2 H_{c}-2 H_{d}-3 H_{Y}\right)^{-1}\left(v_{b}+2 v_{c}-\right. \\
\left.\left.2 v_{d}-3 v_{Y}\right) \mid\right\}\end{array}$ \\
\hline$Q_{3} d_{3}^{c} H_{3}^{d}$ & $\begin{array}{l}n_{33}^{d} \cos \theta_{d} \exp \left\{-\frac{1}{2} \mid\left(-v_{c}-v_{d}+v_{Y}\right)^{T}\left(-H_{c}-H_{d}+H_{Y}\right)^{-1}\left(-v_{d}+v_{Y}\right)+\left(-v_{c}+3 v_{d}+2 v_{Y}\right)^{T}\left(-H_{c}+3 H_{d}+\right.\right. \\
\left.\left.2 H_{Y}\right)^{-1}\left(-v_{c}+3 v_{d}+2 v_{Y}\right)+\left(2 v_{c}-2 v_{d}-3 v_{Y}\right)^{T}\left(2 H_{c}-2 H_{d}-3 H_{Y}\right)^{-1}\left(2 v_{c}-2 v_{d}-3 v_{Y}\right) \mid\right\}\end{array}$ \\
\hline All else & 0 \\
\hline
\end{tabular}

TABLE VI. Electron-type terms.

\begin{tabular}{|c|c|}
\hline Term $L_{i} e_{j}^{c} H_{k}^{d}$ & Coupling $Y_{i j k}^{l}$ \\
\hline$L_{1} e_{2}^{c} H_{3}^{d}$ & $\begin{array}{l}n_{12}^{e} \cos \theta_{d} \exp \left\{-\frac{1}{2} \mid\left(v_{b}-v_{c}+3 v_{d}-3 v_{Y}\right)^{T}\left(H_{b}-H_{c}+3 H_{d}-3 H_{Y}\right)^{-1}\left(v_{b}-v_{c}+3 v_{d}-3 v_{Y}\right)+\left(-v_{b}-v_{c}-v_{d}+\right.\right. \\
\left.\quad 6 v_{Y}\right)^{T}\left(-H_{b}-H_{c}-H_{d}+6 H_{Y}\right)^{-1}\left(-v_{b}-v_{c}-v_{d}+6 v_{Y}\right)+\left(2 v_{c}-2 v_{d}-3 v_{Y}\right)^{T}\left(2 H_{c}-2 H_{d}-3 H_{Y}\right)^{-1}\left(2 v_{c}-2 v_{d}-\right. \\
\left.\left.\quad 3 v_{Y}\right) \mid\right\}\end{array}$ \\
\hline$L_{1} e_{3}^{c} H_{2}^{d}$ & $\begin{array}{l}n_{13}^{e} \sin ?_{d} \sin \theta_{d} \exp \left\{-\frac{1}{2} \mid\left(v_{b}-v_{c}+3 v_{d}-3 v_{Y}\right)^{T}\left(H_{b}-H_{c}+3 H_{d}-3 H_{Y}\right)^{-1}\left(v_{b}-v_{c}+3 v_{d}-3 v_{Y}\right)+\left(-v_{c}-v_{d}+\right.\right. \\
\left.\quad 6 v_{Y}\right)^{T}\left(-H_{c}-H_{d}+6 H_{Y}\right)^{-1}\left(-v_{c}-v_{d}+6 v_{Y}\right)+\left(-v_{b}+2 v_{c}-2 v_{d}-3 v_{Y}\right)^{T}\left(-H_{b}+2 H_{c}-2 H_{d}-3 H_{Y}\right)^{-1}\left(-v_{b}+\right. \\
\left.\left.\quad 2 v_{c}-2 v_{d}-3 v_{Y}\right) \mid\right\}\end{array}$ \\
\hline$L_{2} e_{1}^{c} H_{3}^{d}$ & $\begin{array}{l}n_{21}^{e} \cos \theta_{d} \exp \left\{-\frac{1}{2} \mid\left(-v_{b}-v_{c}+3 v_{d}-3 v_{Y}\right)^{T}\left(-H_{b}-H_{c}+3 H_{d}-3 H_{Y}\right)^{-1}\left(-v_{b}-v_{c}+3 v_{d}-3 v_{Y}\right)+\left(v_{b}-v_{c}-v_{d}+\right.\right. \\
\left.\quad 6 v_{Y}\right)^{T}\left(H_{b}-H_{c}-H_{d}+6 H_{Y}\right)^{-1}\left(v_{b}-v_{c}-v_{d}+6 v_{Y}\right)+\left(2 v_{c}-2 v_{d}-3 v_{Y}\right)^{T}\left(2 H_{c}-2 H_{d}-3 H_{Y}\right)^{-1}\left(2 v_{c}-2 v_{d}-\right. \\
\left.\left.\quad 3 v_{Y}\right) \mid\right\}\end{array}$ \\
\hline$L_{2} e_{3}^{c} H_{1}^{d}$ & $\begin{array}{l}n_{23}^{e} \cos ?_{d} \sin \theta_{d} \exp \left\{-\frac{1}{2} \mid\left(-v_{b}-v_{c}+3 v_{d}-3 v_{Y}\right)^{T}\left(-H_{b}-H_{c}+3 H_{d}-3 H_{Y}\right)^{-1}\left(-v_{b}-v_{c}+3 v_{d}-3 v_{Y}\right)+\left(-v_{c}-v_{d}+\right.\right. \\
\left.\quad 6 v_{Y}\right)^{T}\left(-H_{c}-H_{d}+6 H_{Y}\right)^{-1}\left(-v_{c}-v_{d}+6 v_{Y}\right)+\left(v_{b}+2 v_{c}-2 v_{d}-3 v_{Y}\right)^{T}\left(H_{b}+2 H_{c}-2 H_{d}-3 H_{Y}\right)^{-1}\left(v_{b}+2 v_{c}-\right. \\
\left.\left.\quad 2 v_{d}-3 v_{Y}\right) \mid\right\}\end{array}$ \\
\hline$L_{3} e_{1}^{c} H_{2}^{d}$ & $\begin{array}{l}n_{31}^{e} \sin ?_{d} \sin \theta_{d} \exp \left\{-\frac{1}{2} \mid\left(-v_{c}+3 v_{d}-3 v_{Y}\right)^{T}\left(-H_{c}+3 H_{d}-3 H_{Y}\right)^{-1}\left(-v_{c}+3 v_{d}-3 v_{Y}\right)+\left(v_{b}-v_{c}-v_{d}+6 v_{Y}\right)^{T}\left(H_{b}-\right.\right. \\
\left.\quad H_{c}-H_{d}+6 H_{Y}\right)^{-1}\left(v_{b}-v_{c}-v_{d}+6 v_{Y}\right)+\left(-v_{b}+2 v_{c}-2 v_{d}-3 v_{Y}\right)^{T}\left(-H_{b}+2 H_{c}-2 H_{d}-3 H_{Y}\right)^{-1}\left(-v_{b}+2 v_{c}-\right. \\
\left.\left.\quad 2 v_{d}-3 v_{Y}\right) \mid\right\}\end{array}$ \\
\hline$L_{3} e_{2}^{c} H_{1}^{d}$ & $\begin{array}{l}n_{32}^{e} \cos ?_{d} \sin \theta_{d} \exp \left\{-\frac{1}{2} \mid\left(-v_{c}+3 v_{d}-3 v_{Y}\right)^{T}\left(-H_{c}+3 H_{d}-3 H_{Y}\right)^{-1}\left(-v_{c}+3 v_{d}-3 v_{Y}\right)+\left(-v_{b}-v_{c}-v_{d}+\right.\right. \\
\left.\quad 6 v_{Y}\right)^{T}\left(-H_{b}-H_{c}-H_{d}+H_{Y}\right)^{-1}\left(-v_{b}-v_{c}-v_{d}+6 v_{Y}\right)+\left(v_{b}+2 v_{c}-2 v_{d}-3 v_{Y}\right)^{T}\left(H_{b}+2 H_{c}-2 H_{d}-\right. \\
\left.\left.\quad 3 H_{Y}\right)^{-1}\left(v_{b}+2 v_{c}-2 v_{d}-3 v_{Y}\right) \mid\right\}\end{array}$ \\
\hline$L_{3} e_{3}^{c} H_{3}^{d}$ & $\begin{array}{l}n_{33}^{e} \cos \theta_{d} \exp \left\{-\frac{1}{2} \mid\left(-v_{c}+3 v_{d}-3 v_{Y}\right)^{T}\left(-H_{c}+3 H_{d}-3 H_{Y}\right)^{-1}\left(-v_{c}+3 v_{d}-3 v_{Y}\right)+\left(-v_{c}-v_{d}+6 v_{Y}\right)^{T}\left(-H_{c}-H_{d}+\right.\right. \\
\left.\left.\quad 6 H_{Y}\right)^{-1}\left(-v_{c}-v_{d}+6 v_{Y}\right)+\left(2 v_{c}-2 v_{d}-3 v_{Y}\right)^{T}\left(2 H_{c}-2 H_{d}-3 H_{Y}\right)^{-1}\left(2 v_{c}-2 v_{d}-3 v_{Y}\right) \mid\right\}\end{array}$ \\
\hline All else & \\
\hline
\end{tabular}


TABLE VII. Tabulated numerical values of Moduli.

\begin{tabular}{lccc}
\hline \hline Parameter & Value & Parameter & Value \\
\hline$H_{b 1,1}$ & 1.75219628381272 & $H_{Y 1,1}$ & 0.470254977486118 \\
$H_{b 1,2}$ & -0.735328652705781 & $H_{Y 1,2}$ & 0.701824648617083 \\
$H_{b 1,3}$ & -0.377020719433746 & $H_{Y 1,3}$ & -1.34973735409641 \\
$H_{b 2,2}$ & 1.19315995302413 & $H_{Y 2,2}$ & 0.641604847709697 \\
$H_{b 2,3}$ & $H_{Y 2,3}$ & 0.108762493856499 \\
$H_{c 1,1}$ & 0.675543994721913 & $v_{c 1}$ & -0.250519055696569 \\
$H_{c 1,2}$ & 1.41562893856031 & $v_{c 2}$ & -1.4656911323334 \\
$H_{c 1,3}$ & 1.05931181064608 & $v_{c 3}$ & -0.405862359379641 \\
$H_{c 2,2}$ & $v_{Y 1}$ & -0.417353540440179 \\
$H_{c 2,3}$ & 1.45371284442019 & $v_{Y 2}$ & 0.688596965400472 \\
$H_{d 1,1}$ & $v_{Y 3}$ & -1.50894939341168 \\
$H_{d 1,2}$ & $\phi_{1}$ & 0.439157499240369 \\
$H_{d 1,3}$ & $\theta_{1}$ & -1.37741142245986 \\
$H_{d 2,2}$ & 0.509312354295455 & $\phi_{2}$ & 3.76691301205782 \\
$H_{d 2,3}$ & -1.11688627765089 & $\theta_{2}$ & 6.06985994999668 \\
$\Lambda_{0}$ & -1.13434995566124 & $\ldots$ & $\ldots$ \\
\hline \hline
\end{tabular}

[1] B. S. Acharya and E. Witten, arXiv:hep-th/0109152.

[2] B. S. Acharya, arXiv:hep-th/0011089.

[3] B. S. Acharya, K. Bobkov, G. L. Kane, P. Kumar, and J. Shao, Phys. Rev. D 76, 126010 (2007).

[4] B. S. Acharya, K. Bobkov, G. L. Kane, J. Shao, and P. Kumar, Phys. Rev. D 78, 065038 (2008).

[5] G. Papadopoulos and P. K. Townsend, Phys. Lett. B 357, 300 (1995).

[6] G. Kane and M. W. Winkler, J. Cosmol. Astropart. Phys. 02 (2020) 019.

[7] B. S. Acharya, K. Bobkov, and P. Kumar, J. High Energy Phys. 11 (2010) 105.

[8] G. Kane and M. W. Winkler, Phys. Rev. D 100, 066005 (2019).

[9] B. S. Acharya and S. Gukov, Phys. Rep. 392, 121 (2004).

[10] J. L. Bourjaily and S. Espahbodi, arXiv:0804.1132.

[11] J. L. Bourjaily, arXiv:0901.3785.

[12] F. Marchesano, D. Regalado, and G. Zoccarato, J. High Energy Phys. 04 (2015) 179.

[13] R. Tatar and T. Watari, Nucl. Phys. B747, 212 (2006).

[14] C. Beasley, J. J. Heckman, and C. Vafa, J. High Energy Phys. 01 (2009) 059.

[15] T. Pantev and M. Wijnholt, J. Geom. Phys. 61, 1223 (2011).

[16] A. P. Braun, S. Cizel, M. Hbner, and S. Schfer-Nameki, J. High Energy Phys. 03 (2019) 199.

[17] A. Kovalev, J. Reine Angew. Math. 565 (2003).

[18] A. P. Braun and M. Del Zotto, J. High Energy Phys. 05 (2017) 080.

[19] A. P. Braun and S. Schfer-Nameki, J. High Energy Phys. 04 (2018) 126.

[20] A. Corti, M. Haskins, J. Nordstrm, and T. Pacini, Duke Math. J. 164, 1971 (2015).
[21] A. Kennon, arXiv:1810.12659.

[22] J. Halverson and D. R. Morrison, J. High Energy Phys. 04 (2016) 100.

[23] A. Sen, J. High Energy Phys. 09 (1997) 001.

[24] M. Atiyah and E. Witten, Adv. Theor. Math. Phys. 6, 1 (2002).

[25] P. Berglund and A. Brandhuber, Nucl. Phys. B641, 351 (2002).

[26] P. Du Val, Math. Proc. Cambridge Philos. Soc. 30, 453459 (1934).

[27] P. B. Kronheimer, J. Diff. Geom. 29, 665 (1989).

[28] S. H. Katz and C. Vafa, Nucl. Phys. B497, 146 (1997).

[29] S. Katz and D. Morrison, J. Alg. Geom. 1, 449 (1992).

[30] H. Ooguri and C. Vafa, Nucl. Phys. B766, 2133 (2007).

[31] C. H. Clemens and S. Raby, J. High Energy Phys. 04 (2020) 059.

[32] J. L. Bourjaily, Phys. Rev. D 76, 046004 (2007).

[33] E. Dudas and E. Palti, J. High Energy Phys. 01 (2010) 127.

[34] N. Cabo Bizet, A. Klemm, and D. Vieira Lopes, arXiv: 1404.7645.

[35] H. Godazgar, M. Godazgar, and M. J. Perry, J. High Energy Phys. 06 (2013) 044.

[36] J. Evslin, J. High Energy Phys. 08 (2004) 021.

[37] E. Palti, J. High Energy Phys. 07 (2012) 065.

[38] D. Gaiotto, G. W. Moore, and E. Witten, arXiv:1506 .04087.

[39] J. A. Harvey and G. W. Moore, arXiv:hep-th/9907026.

[40] C. Beasley and E. Witten, J. High Energy Phys. 10 (2003) 065.

[41] S. A. R. Ellis, G. L. Kane, and B. Zheng, J. High Energy Phys. 07 (2015) 081.

[42] J. A. Casas and C. Munoz, Phys. Lett. B 306, 288 (1993). 
[43] B. S. Acharya, K. Boek, M. Crispim Romo, S. F. King, and C. Pongkitivanichkul, J. High Energy Phys. 11 (2016) 173.

[44] K. S. Babu, B. Bajc, and S. Saad, J. High Energy Phys. 02 (2017) 136.

[45] G. Ross and M. Serna, Phys. Lett. B 664, 97 (2008).
[46] P. Anastasopoulos, M. Bianchi, E. Dudas, and E. Kiritsis, J. High Energy Phys. 11 (2006) 057.

[47] B. S. Acharya and K. Bobkov, J. High Energy Phys. 09 (2010) 001.

[48] B. Acharya and E. Witten, Chiral fermions from manifolds of G(2) holonomy, arXiv:hep-th/0109152. 